\title{
$T$ duality constraint on R-R couplings
}

\author{
Mohammad R. Garousi॰* \\ Department of Physics, Faculty of Science, Ferdowsi University of Mashhad, P.O. Box 1436, Mashhad, Iran
}

(Received 14 September 2020; accepted 7 October 2020; published 21 October 2020)

\begin{abstract}
It has been speculated that the metric, $B$-field, and dilaton couplings in the low energy effective action of string theory at any order of $\alpha^{\prime}$ may be found by imposing the gauge symmetries and the $T$ duality on the effective action. This proposal can be extended to include the Ramond-Ramond (R-R) couplings as well. In this paper, we first find the dimensional reduction of the R-R fields and then perform explicitly the $T$ duality constraint on the R-R couplings at the supergravity level. Up to an overall factor, it reproduces the democratic form of the R-R couplings.
\end{abstract}

DOI: 10.1103/PhysRevD.102.086019

One of the most exciting discoveries in perturbative string theory is $T$ duality [1-3], which appears when one compactifies theory on a torus. It has been speculated that the invariance of the effective action of bosonic string theory under the standard gauge transformations and under nonstandard $T$ duality transformations which receive $\alpha^{\prime}$ corrections, may be used as constraints to construct the low energy effective action of the string theory [4]. In this approach, using the field redefinitions freedom [5], one first constructs the minimum number of gauge invariant couplings. Then one reduces them on a circle. The reduced actions must be invariant under the standard Buscher rules $[6,7]$ plus their $\alpha^{\prime}$ corrections [8-11]. Using this approach, the effective actions of the bosonic string theory up to order $\alpha^{3}$ have been found in Refs. [11,12]. The NS-NS part of the effective action of the superstring theory on a manifold with boundary at the leading order of $\alpha^{\prime}$ has been also found in Ref. [13] by imposing gauge symmetries, the $T$ duality and by imposing the $S$ duality, another duality which exists in the superstring theory [3]. In particular, the well known Gibbons-Hawking-York term $[14,15]$ has been dictated by the string dualities [13].

Another $T$ duality based approach for constructing the $D$-dimensional effective action is the double field theory [16-20] in which the effective action in $2 \mathrm{D}$ space is constrained to be invariant under $T$ duality and under gauge transformations. The $T$ duality in this case, however, is the standard $O(D, D)$ transformation without $\alpha^{\prime}$ corrections, whereas the gauge transformation is nonstandard, which receives $\alpha^{\prime}$ corrections [20-23]. This approach has

\footnotetext{
"garousi@um.ac.ir
}

Published by the American Physical Society under the terms of the Creative Commons Attribution 4.0 International license. Further distribution of this work must maintain attribution to the author(s) and the published article's title, journal citation, and DOI. Funded by SCOAP ${ }^{3}$. been extended in Ref. [24] to type II superstring theories. These $T$ duality approaches for constructing the effective actions are based on the observation made by Sen in the context of closed string field theory [25] that the effective action of string theory should be invariant under $T$ duality to all orders in $\alpha^{\prime}$.

In this paper we would like to extend the first approach to the couplings in type II superstring theories. The type II string theories have NS-NS fields which are the same as the fields in the bosonic string theory, as well as some R-R fields which are also bosonic fields. The $D_{p}$-branes in type II string theories carry the R-R charges [26]. These theories have also NS-R and R-NS femionic fields in which we are not interested. The odd-form R-R potentials appear in type IIA and even-forms appear in type IIB. It is known that the compactification of type IIA theory on a circle transforms to the compactification of type IIB theory on another circle under the $T$ duality transformations. To study the effective action of the bosonic fields in these theories, it is convenient to collect the two theories to one theory which is called type II theory. It has both odd- and even-form R-R potentials. When compactifying this theory on a circle, the effective action then is expected to be invariant under the $T$ duality transformations, as in the bosonic string theory.

When compactifying string theory on a circle with unit radius and with the killing coordinate $y$, the $T$ duality transformations for the NS-NS fields are the Buscher rules $[6,7]$, i.e.,

$$
\begin{array}{rlrl}
e^{2 \phi^{\prime}} & =\frac{e^{2 \phi}}{G_{y y}} ; & G_{y y}^{\prime}=\frac{1}{G_{y y}}, \\
G_{\mu y}^{\prime}=\frac{B_{\mu y}}{G_{y y}} ; & G_{\mu \nu}^{\prime}=G_{\mu \nu}-\frac{G_{\mu y} G_{\nu y}-B_{\mu y} B_{\nu y}}{G_{y y}}, \\
B_{\mu y}^{\prime}=\frac{G_{\mu y}}{G_{y y}} ; & B_{\mu \nu}^{\prime}=B_{\mu \nu}-\frac{B_{\mu y} G_{\nu y}-G_{\mu y} B_{\nu y}}{G_{y y}},
\end{array}
$$


where $\mu, \nu$ denote any direction other than $y$. In above transformations the metric is in the string frame. If one assumes fields are transformed covariantly under the coordinate transformations, then the above transformations receive $\alpha^{\prime}$ corrections [8-11]. The $T$ duality transformations of the R-R fields at the leading order of $\alpha^{\prime}$ have been found in Ref. [27], i.e.,

$$
\begin{aligned}
& C_{\mu \cdots \nu \alpha y}^{(n)}=C_{\mu \cdots \nu \alpha}^{(n-1)}-\frac{C_{[\mu \cdots \nu \mid y}^{(n-1)} G_{\mid \alpha] y}}{G_{y y}}, \\
& C_{\mu \cdots \nu \alpha \beta}^{\prime(n)}=C_{\mu \cdots \nu \alpha \beta y}^{(n+1)}+C_{[\mu \cdots \nu \alpha}^{(n-1)} B_{\beta] y}+\frac{C_{[\mu \cdots \nu \mid y}^{(n-1)} B_{|\alpha| y} G_{\mid \beta] y}}{G_{y y}} .
\end{aligned}
$$

They may also have $\alpha^{\prime}$ corrections in which we are not interested in this paper. Our notation for making antisymmetry is such that, e.g., $C_{\left[\mu_{1} \mu_{2}\right.}^{(2)} B_{\left.\mu_{3}\right] y}=C_{\mu_{1} \mu_{2}}^{(2)} B_{\mu_{3} y}+$ $C_{\mu_{2} \mu_{3}}^{(2)} B_{\mu_{1} y}-C_{\mu_{1} \mu_{3}}^{(2)} B_{\mu_{2} y}$. The $T$ duality transformations (1) and (2) are such that they are consistent with the fact that $D_{p}$-brane in type II theory transform to $D_{p-1}$-brane or $D_{p+1}$-brane depending on whether the brane is along or orthogonal to the circle on which the $T$ duality is imposed. In fact the R-R fields couple to the $D_{p}$-brane as

$$
\int_{M^{p+1}} e^{B} C
$$

where $C=\sum_{n=0}^{8} C^{(n)}$. It is invariant under the R-R gauge transformation $\delta C=d \Lambda+H \Lambda$, where $\Lambda=\sum_{n=0}^{7} \Lambda^{(n)}$. The $T$ duality transformations (1) and (2) produce the following transformations:

$$
\left(e^{B} C\right)_{\ldots y}^{\prime}=\left(e^{B} C\right)_{\ldots} ; \quad\left(e^{B} C\right)_{\ldots .}^{\prime}=\left(e^{B} C\right)_{\ldots y},
$$

where dots represent some world-volume indices. In other words, the coupling (3) is covariant under the $T$ duality transformations.

The effective action of type II string theory on the closed manifolds at the leading order of $\alpha^{\prime}$ is the well-known type II supergravity (see, e.g., Ref. [3]). The first higher derivative corrections to this action is at order $\alpha^{\prime 3}$. The Riemann curvature couplings at this order are known in the literature [28-30]. There are many other couplings involving $B$-field, dilaton, and R-R fields at this order. Some of them have been found in Refs. [31-33]. There are also boundary terms at this order when manifolds have boundaries which are not known in the literature. We expect all these couplings might be found by imposing the appropriate gauge transformations and string duality constraints on the effective action. In fact, the known Riemann curvature couplings are reproduced by this method in Ref. [34]. In this paper we present the technical details for imposing the $T$ duality constraint to reproduce the R-R couplings at the supergravity level and leave the calculations at order $\alpha^{\prime 3}$ for the future works. The NS-NS couplings at the leading order of $\alpha^{\prime}$ are the same as the corresponding couplings in the bosonic theory.

The R-R couplings, as in Eq. (3), should be invariant under the R-R gauge transformations. The R-R couplings in the effective action should be in terms of the R-R field strength, i.e.,

$$
F^{(n)}=d C^{(n-1)}+H \wedge C^{(n-3)},
$$

which is invariant under the R-R gauge transformations. ${ }^{1}$ Since $F^{(n)}$ is not the exterior derivative of an $(n-1)$ form for $n>2$, it satisfies the following anomalous Bianchi identity:

$$
d F^{(n)}=-H \wedge F^{(n-2)} .
$$

At the two-derivative level, the gauge invariance constraint then requires the following couplings in the string frame:

$$
\begin{aligned}
& \mathbf{S}_{0}^{\mathrm{R}-\mathrm{R}}=-\frac{2}{\kappa^{2}} \int d^{10} x \sqrt{-G} \sum_{n=1}^{9} a_{n}\left|F^{(n)}\right|_{G}^{2} \\
& =-\frac{2}{\kappa^{2}} \int d^{10} x \sqrt{-G} \sum_{n=1}^{9} \frac{a_{n}}{n !} G^{a_{1} b_{1}} \ldots G^{a_{n} b_{n}} F_{a_{1} \cdots a_{n}}^{(n)} F_{b_{1} \cdots b_{n}}^{(n)},
\end{aligned}
$$

where $a_{1}, a_{2}, \ldots, a_{9}$ are 9 parameters that the R-R gauge symmetry cannot fix. Since the R-R field strengths (5) are nonlinear for $n>2$, these constants cannot be absorbed by the normalization of the R-R potentials. We are going to show that they all can be written in terms of $a_{1}$ by imposing on them the $T$ duality constraint as well. We did not include $F^{(0)}$ and $F^{(10)}$ terms in the above couplings because they do not include dynamical fields. In writing the above couplings we assume the R-R fields are all independent. The on-shell physics, however, requires not all components of $C^{(4)}$ to be independent. Moreover, the fields $C^{(5)}, C^{(6)}, C^{(7)}, C^{(8)}$ are not independent. That means in the equations of motion one has to impose some extra constraints on the R-R field strengths to have physical fields in the equations of motion.

To impose the $T$ duality constraint on the effective action (7), we have to consider the Kaluza-Klein reduction of the 10-dimensional metric, i.e.,

\footnotetext{
${ }^{1}$ The definition of the R-R field strength for $n=5$ is slightly different from the common definition in the supergravity literature (see, e.g., Ref. [3]). However, the field redefinition of the R-R potentials $C^{(4)} \rightarrow C^{(4)}+\frac{1}{2} B \wedge C^{(2)}$ and $C^{(2)} \rightarrow-C^{(2)}$ transforms the above $F^{(5)}$ to the standard form of $F^{(5)}=$ $d C^{(4)}-\frac{1}{2} H \wedge C^{(2)}+\frac{1}{2} B \wedge d C^{(2)}$.
} 


$$
d s^{2}=\bar{g}_{\mu \nu} d x^{\mu} d x^{\nu}+e^{\varphi}\left(d y+g_{\mu} d x^{\mu}\right)^{2},
$$

which is invariant under $y$-coordinate transformation $y \rightarrow$ $y-\lambda(x)$ and the gauge transformation $g_{\mu} \rightarrow g_{\mu}+\partial_{\mu} \lambda$. Apart from this $U(1)$ isometry gauge symmetry, the reduced theory should have another $U(1)$ gauge symmetry corresponding to the Kalb-Ramond form $B=\frac{1}{2} B_{\mu \nu} d x^{\mu} \wedge$ $d x^{\nu}+B_{\mu y} d x^{\mu} \wedge d y$. The reduction of any theory containing the $B$ field is manifestly the $U(1) \times U(1)$ gauge invariant if one uses the following reduction for the $B$ field and dilaton as well [35]:

$$
\begin{aligned}
G_{a b} & =\left(\begin{array}{cc}
\bar{g}_{\mu \nu}+e^{\varphi} g_{\mu} g_{\nu} & e^{\varphi} g_{\mu} \\
e^{\varphi} g_{\nu} & e^{\varphi}
\end{array}\right), \\
B_{a b} & =\left(\begin{array}{cc}
\bar{b}_{\mu \nu}+\frac{1}{2} b_{[\mu} g_{\nu]} & b_{\mu} \\
-b_{\nu} & 0
\end{array}\right), \\
\Phi & =\bar{\phi}+\varphi / 4,
\end{aligned}
$$

where $\bar{g}_{\mu \nu}, \bar{b}_{\mu \nu}, \bar{\phi}$ are the metric, a two-form, and the dilaton, respectively, in the base space, and $g_{\mu}, b_{\mu}$ are two vectors in this space. The inverse of the above 10-dimensional metric is

$$
G^{a b}=\left(\begin{array}{cc}
\bar{g}^{\mu \nu} & -g^{\mu} \\
-g^{\nu} & e^{-\varphi}+g_{\alpha} g^{\alpha}
\end{array}\right),
$$

where $\bar{g}^{\mu \nu}$ is the inverse of the base space metric which raises the index of the vectors. The Buscher rules (1) in the parametrizations (9) become the following linear transformations:

$$
\begin{array}{clc}
\varphi^{\prime}=-\varphi, & g_{\mu}^{\prime}=b_{\mu}, & b_{\mu}^{\prime}=g_{\mu}, \\
\bar{g}_{\alpha \beta}^{\prime}=\bar{g}_{\alpha \beta}, & \bar{b}_{\alpha \beta}^{\prime}=\bar{b}_{\alpha \beta}, & \bar{\phi}^{\prime}=\bar{\phi} .
\end{array}
$$

They form a $Z_{2}$ group, i.e., $\left(x^{\prime}\right)^{\prime}=x$, where $x$ is any field in the base space. These transformations receive higher derivative corrections in which we are not interested in this paper.

The reduction of field strength of the $B$ field in the parametrizations (9) becomes

$$
\begin{aligned}
& H_{\mu \nu \alpha}=\bar{H}_{\mu \nu \alpha}+g_{[\mu} W_{\nu \alpha]}, \\
& H_{\mu \nu y}=W_{\mu \nu},
\end{aligned}
$$

where $W$ is field strength of the $U(1)$ gauge field $b_{\mu}$, i.e., $W=d b$, and the three-form $\bar{H}$ which is torsion in the base space, is defined as

$$
\bar{H}_{\mu \nu \alpha} \equiv \hat{H}_{\mu \nu \alpha}-\frac{1}{2} g_{[\mu} W_{\nu \alpha]}-\frac{1}{2} b_{[\mu} V_{\nu \alpha]},
$$

where $\hat{H}$ is field strength of the two-form $\bar{b}_{\mu \nu}$ and $V$ is field strength of the $U(1)$ gauge field $g_{\mu}$, i.e., $V=d g$. The three-form $\bar{H}$ is invariant under the $T$ duality and under various gauge transformations, e.g., under the $U(1)$ isometry gauge transformation, the $B_{\mu \nu}$ components of the $B$ field transform as $B_{\mu \nu} \rightarrow B_{\mu \nu}+b_{\mu} \partial_{\nu} \lambda-b_{\nu} \partial_{\mu} \lambda$. Hence, $H_{\mu \nu \alpha} \rightarrow H_{\mu \nu \alpha}+\partial_{[\mu} \lambda W_{\nu \alpha]}$. The anomalous term is canceled with the transformation of the last term in the first line of Eq. (12). Hence, $\bar{H}_{\mu \nu \alpha}$ remains invariant. It is also obvious from Eq. (12) that $\bar{H}$ is invariant under the $B$-field gauge transformation $B_{a b} \rightarrow B_{a b}+\partial_{a} \omega_{b}-\partial_{b} \omega_{a}$. Since $\bar{H}$ is not the exterior derivative of a two form, it satisfies the following anomalous Bianchi identity:

$$
\partial_{[\mu} \bar{H}_{\nu \alpha \beta]}=-V_{[\mu \nu} W_{\alpha \beta]} .
$$

Using the reduction (9), one finds that the reduction of any 10 -dimensional gauge invariant coupling can be written in terms of gauge invariant tensors $\bar{R}, \bar{H}, V, W, \bar{\phi}, \varphi$ and their derivatives. Hence, the reduction is consistent with the $U(1) \times U(1)$ gauge symmetry [35]. In this paper, among other things, we are going to find such reduction for the R-R fields.

To find such reduction for the R-R fields, we first note that in the parametrization (9) the nonlinear $T$ duality transformations of the R-R fields (2) become

$$
\begin{aligned}
& C_{\mu \cdots \nu \alpha y}^{\prime(n)}=C_{\mu \cdots \nu \alpha}^{(n-1)}-C_{[\mu \cdots \nu \mid y}^{(n-1)} g_{\alpha]}, \\
& C_{\mu \cdots \nu \alpha \beta}^{\prime(n)}=C_{\mu \cdots \nu \alpha \beta y}^{(n+1)}+C_{[\mu \cdots \nu \alpha}^{(n-1)} b_{\beta]}+C_{[\mu \cdots \nu \mid y}^{(n-1)} b_{\alpha} g_{\beta]},
\end{aligned}
$$

which are still nonlinear. On the right-hand side the $\mathrm{R}-\mathrm{R}$ fields are 10-dimensional whereas the $b_{\alpha}, g_{\alpha}$ are 9-dimensional fields. To proceed further then one has to reduce the R-R fields as well. The reduction should be consistent with the $U(1) \times U(1)$ gauge symmetry.

We consider the following reductions for the R-R fields:

$$
\begin{aligned}
C_{\mu_{1} \cdots \mu_{n}}^{(n)} & =\bar{c}_{\mu_{1} \cdots \mu_{n}}^{(n)}+\bar{c}_{\left[\mu_{1} \cdots \mu_{n-1}\right.}^{(n-1)} g_{\left.\mu_{n}\right]}, \\
C_{\mu_{1} \cdots \mu_{n} y}^{(n+1)} & =\bar{c}_{\mu_{1} \cdots \mu_{n}}^{(n)},
\end{aligned}
$$

where $\bar{c}^{(n)}$ are R-R potentials in the 9-dimensional base space. The nonlinear $T$ duality transformations (2) in the above parametrizations become the following linear transformations:

$$
\bar{c}_{\mu_{1} \cdots \mu_{n}}^{\prime(n)}=\bar{c}_{\mu_{1} \cdots \mu_{n}}^{(n)}
$$

It is remarkable that in the parametrizations (16), the 9-dimensional R-R fields become invariant under the $T$ duality transformation. They may, however, receive corrections at the higher order of $\alpha^{\prime}$ in which we are not interested in this paper. 
The reduction of the 10-dimensional R-R field strength in the parametrizations (16) becomes

$$
\begin{aligned}
F_{\mu_{1} \ldots \mu_{n}}^{(n)} & =F_{\mu_{1} \ldots \mu_{n}}^{V(n)}+g_{\left[\mu_{1}\right.} F_{\left.\mu_{2} \cdots \mu_{n}\right]}^{W(n-1)}, \\
F_{\mu_{1} \ldots \mu_{n-1} y}^{(n)} & =F_{\mu_{1} \ldots \mu_{n-1}}^{W(n-1)} .
\end{aligned}
$$

The forms $F^{W(n)}$ and $F^{V(n)}$ are defined as

$$
\begin{aligned}
F^{W(n)} & \equiv \bar{F}^{(n)}+\bar{H} \wedge \bar{c}^{(n-3)}+(-1)^{(n-2)} \bar{c}^{(n-2)} \wedge W, \\
F^{V(n)} & \equiv \bar{F}^{(n)}+\bar{H} \wedge \bar{c}^{(n-3)}+(-1)^{(n-2)} \bar{c}^{(n-2)} \wedge V,
\end{aligned}
$$

where $\bar{F}^{(n)}$ is field strength of the 9-dimensional R-R potential $\bar{c}^{(n-1)}$, i.e., $\bar{F}^{(n)}=d \bar{c}^{(n-1)}$. These forms are invariant under various gauge transformations, e.g., under the $U(1)$ isometry gauge transformation, the $C_{\mu_{1} \cdots \mu_{n}}^{(n)}$ components of the R-R $n$-form potential $C^{(n)}=$ $\frac{1}{n} C_{\mu_{1} \cdots \mu_{n}}^{(n)} d x^{\mu_{1}} \cdots \wedge d x^{\mu_{n}}+C_{\mu_{1} \cdots \mu_{n-1} y}^{(n)} d x^{\mu_{1}} \cdots \wedge d x^{\mu_{n-1}} \wedge d y$ transform as $C_{\mu_{1} \cdots \mu_{n}}^{(n)} \rightarrow C_{\mu_{1} \cdots \mu_{n}}^{(n)}+C_{\left[\mu_{1} \cdots \mu_{n-1}\right.}^{(n-1)} \partial_{\left.\mu_{n}\right]} \lambda$. Then one can show that $F_{\mu_{1} \cdots \mu_{n}}^{(n)} \rightarrow F_{\mu_{1} \cdots \mu_{n}}^{(n)}+\partial_{\left[\mu_{1}\right.} \lambda F_{\left.\mu_{2} \cdots \mu_{n}\right]}^{W(n-1)}$ where we have used the fact that $g \wedge g$ and its gauge transformation are zero. The anomalous term is canceled with the transformation of the last term in the first line of Eq. (18). Therefore, $F^{V}$ is invariant under the $U(1)$ isometry gauge transformation. Since $C_{\mu_{1} \cdots \mu_{n-1} y}^{(n)}$ components of the R-R potential are invariant under the $U(1)$ gauge transformation, the second relation in (18) indicates that $F^{W}$ is also invariant. It is also obvious from Eq. (18) that $F^{V}, F^{W}$ are invariant under the R-R gauge transformations because the left-hand sides are invariant. Since the gauge invariant $n$ forms $F^{V(n)}, F^{W(n)}$ are not exterior derivative of any $(n-1)$-form, their corresponding Bianchi identities are anomalous. The anomalous Bianchi identities are

$$
\begin{aligned}
d F^{V(n)} & =(-1)^{(n-2)} F^{W(n-1)} \wedge V-\bar{H} \wedge F^{V(n-2)}, \\
d F^{W(n)} & =(-1)^{(n-2)} F^{V(n-1)} \wedge W-\bar{H} \wedge F^{W(n-2)},
\end{aligned}
$$

where we have also used the anomalous Bianchi identity (14) and the fact that $\bar{H} \wedge \bar{H}=0$. Under the $T$ duality, the gauge invariant forms (19) transform as

$$
F^{W(n)} \leftrightarrow F^{V(n)} .
$$

One expects the reduction of any 10-dimensional coupling involving the R-R field strength and its higher derivatives should be written in terms of 9-dimensional gauge invariant fields $\bar{R}, \bar{H}, V, W, F^{V(n)}, F^{W(n)}, \bar{\phi}, \varphi$ and their covariant derivatives.

Using the reductions (9), (10), and (16), it is straightforward to reduce different terms in Eq. (7). The reduction of $\sqrt{-G}$ and the R-R coupling $\left|F^{(1)}\right|_{G}^{2},\left|F^{(2)}\right|_{G}^{2}$, and $\left|F^{(3)}\right|_{G}^{2}$ are the following:

$$
\begin{aligned}
\sqrt{-G}= & e^{\varphi / 2} \sqrt{-\bar{g}}, \\
\left|F^{(1)}\right|_{G}^{2}= & e^{-\varphi / 2}\left(e^{\varphi / 2}\left|\bar{F}^{(1)}\right|_{\bar{g}}^{2}\right), \\
\left|F^{(2)}\right|_{G}^{2}= & \left.\left.e^{-\varphi / 2}\left(e^{-\varphi / 2}\left|\bar{F}^{(1)}\right|_{\bar{g}}^{2}+e^{\varphi / 2} \mid \bar{F}^{(2)}+\bar{c}^{(0)} V\right)\right|_{\bar{g}} ^{2}\right), \\
\left|F^{(3)}\right|_{G}^{2}= & e^{-\varphi / 2}\left(e^{-\varphi / 2}\left|\bar{F}^{(2)}+\bar{c}^{(0)} W\right|_{\bar{g}}^{2}+e^{\varphi / 2} \mid \bar{F}^{(3)}\right. \\
& \left.+\bar{H} \bar{c}^{(0)}-\left.\bar{c}^{(1)} \wedge V\right|_{\bar{g}} ^{2}\right) .
\end{aligned}
$$

The subscript $\bar{g}$ in $|\cdots|_{\bar{g}}^{2}$ indicates that the indices are contracted with the base space metric $\bar{g}^{\mu \nu}$. The reduction of $\left|F^{(n)}\right|_{G}^{2}$ for $n>3$ can be written as

$$
\left|F^{(n)}\right|_{G}^{2}=e^{-\varphi / 2}\left(e^{-\varphi / 2}\left|F^{W(n-1)}\right|_{\bar{g}}^{2}+e^{\varphi / 2}\left|F^{V(n)}\right|_{\bar{g}}^{2}\right) .
$$

As expected, the nongauge invariant term in Eq. (18) is canceled in the reduction of 10-dimensional couplings involving the R-R field strength. However, it has gauge invariant contribution to the reduction of couplings which involve derivatives of the R-R field strength in which we are not interested in this paper.

Using the fact that the nondynamical field strength $F^{(10)}$ in the 10-dimensional spacetime has been ignored, one should also ignore the nondynamical fields in the 9-dimensional base space. Hence the reduction of $\left|F^{(9)}\right|_{G}^{2}$ becomes

$$
\left|F^{(9)}\right|_{G}^{2}=e^{-\varphi}\left|F^{W(8)}\right|_{\bar{g}}^{2} .
$$

Having found the reduction of the R-R fields, we now impose the $T$ duality constraint on the effective action (7) to fix the parameters $a_{1}, \ldots, a_{9}$. According to this proposal, the effective action should satisfy the following relation:

$$
S_{\text {eff }}(\psi)-S_{\text {eff }}\left(\psi^{\prime}\right)=\mathrm{TD}
$$

where $S_{\text {eff }}$ is the reduction of the 10-dimensional action on the circle, $\psi$ represents all massless fields in the base space, and $\psi^{\prime}$ represents their transformations under the $T$ duality transformations (11) and (17). On the right-hand side, TD represents some total derivative terms in the base space, which become zero if the base space has no boundary. They should be reproduced by the boundary action if the base space has boundary [13].

Using the reduction (23), one finds the reduction of the effective action (7) becomes

$$
\begin{aligned}
S_{\mathrm{eff}}^{\mathrm{R}-\mathrm{R}}(\psi)= & -\frac{4 \pi}{\kappa^{2}} \int d^{9} x \sqrt{-\bar{g}}\left[\sum_{n=1}^{8} a_{n} \frac{e^{\varphi / 2}}{n !}\left|F^{V(n)}\right|_{\bar{g}}^{2}\right. \\
& \left.+\sum_{n=1}^{8} a_{n+1} \frac{e^{-\varphi / 2}}{n !}\left|F^{W(n)}\right|_{\bar{g}}^{2}\right] .
\end{aligned}
$$

Under the $T$ duality transformations (11) and (21), it becomes 


$$
\begin{aligned}
S_{\mathrm{eff}}^{\mathrm{R}-\mathrm{R}}\left(\psi^{\prime}\right)= & -\frac{4 \pi}{\kappa^{2}} \int d^{9} x \sqrt{-\bar{g}}\left[\sum_{n=1}^{8} a_{n} \frac{e^{-\varphi / 2}}{n !}\left|F^{W(n)}\right|_{\bar{g}}^{2}\right. \\
& \left.+\sum_{n=1}^{8} a_{n+1} \frac{e^{\varphi / 2}}{n !}\left|F^{V(n)}\right|_{\bar{g}}^{2}\right] .
\end{aligned}
$$

One can easily observe that the effective action (7) satisfies the constraint (25) with zero total derivative terms on the right-hand side provided that there is the following recursion relation between the parameters:

$$
a_{n}=a_{n+1}
$$

Hence the $T$ duality constraint fixes all 9 parameters in Eq. (7) in terms of one normalization parameter $a_{1}$. Since there is no total derivative terms on the right-hand side of the $T$ duality constraint (25) in this case, the boundary action has no R-R couplings at the leading order of $\alpha^{\prime}$, as expected. The $T$ duality constraint on the NS-NS couplings, however, satisfies the relation (25) with some total derivative terms on the right-hand side which can be canceled by the Gibbons-Hawking-York boundary term as well as another boundary term which is not consistent with the $S$ duality [13]. The duality constraints on the NS-NS couplings also reproduce the standard bulk couplings [13].
Therefore, the gauge symmetry and the $T$ duality fix the low energy effective action of type II string theory in closed spacetime manifold to be

$$
\begin{aligned}
\mathbf{S}_{0}= & -\frac{2}{\kappa^{2}} \int d^{10} x \sqrt{-G}\left[e^{-2 \Phi}\left(R+4 \nabla_{a} \Phi \nabla^{a} \Phi-\frac{1}{12} H^{2}\right)\right. \\
& \left.+a_{1} \sum_{n=1}^{9}\left|F^{(n)}\right|_{G}^{2}\right] .
\end{aligned}
$$

Up to the overall factor $a_{1}$, the R-R couplings are the democratic R-R couplings that have been found in Refs. [24,36]. The parameter $a_{1}$ can be absorbed by the normalization of the $\mathrm{R}-\mathrm{R}$ potentials. When spacetime has boundary, the duality constraint dictates that the GibbonsHawking-York boundary term must be also added to the above action. We expect the $\alpha^{3}$ corrections to the action (29) can also be found by imposing on the effective action the gauge symmetries as well as the string dualities. We leave the details of these calculations for future works.

\section{ACKNOWLEDGMENTS}

This work is supported by Ferdowsi University of Mashhad.
[1] A. Giveon, M. Porrati, and E. Rabinovici, Phys. Rep. 244, 77 (1994).

[2] E. Alvarez, L. Alvarez-Gaume, and Y. Lozano, Nucl. Phys. B, Proc. Suppl. 41, 1 (1995).

[3] K. Becker, M. Becker, and J. H. Schwarz, String Theory and M-theory: A Modern Introduction (Cambridge University Press, 2007).

[4] M. R. Garousi, Phys. Rep. 702, 1 (2017).

[5] R. R. Metsaev and A. A. Tseytlin, Nucl. Phys. B293, 385 (1987).

[6] T. H. Buscher, Phys. Lett. B 194, 59 (1987).

[7] T. H. Buscher, Phys. Lett. B 201, 466 (1988).

[8] A. A. Tseytlin, Mod. Phys. Lett. A 06, 1721 (1991).

[9] E. Bergshoeff, B. Janssen, and T. Ortin, Classical Quantum Gravity 13, 321 (1996).

[10] N. Kaloper and K. A. Meissner, Phys. Rev. D 56, 7940 (1997).

[11] M. R. Garousi, Phys. Rev. D 99, 126005 (2019).

[12] M. R. Garousi, Eur. Phys. J. C 79, 827 (2019).

[13] M. R. Garousi, Phys. Lett. B 809, 135733 (2020).

[14] J. W. York, Jr., Phys. Rev. Lett. 28, 1082 (1972).

[15] G. W. Gibbons and S. W. Hawking, Phys. Rev. D 15, 2752 (1977).

[16] W. Siegel, Phys. Rev. D 47, 5453 (1993).

[17] W. Siegel, Phys. Rev. D 48, 2826 (1993).

[18] W. Siegel, arXiv:hep-th/9308133.

[19] C. Hull and B. Zwiebach, J. High Energy Phys. 09 (2009) 099.
[20] G. Aldazabal, D. Marques, and C. Nunez, Classical Quantum Gravity 30, 163001 (2013).

[21] O. Hohm, C. Hull, and B. Zwiebach, J. High Energy Phys. 08 (2010) 008.

[22] O. Hohm and B. Zwiebach, J. High Energy Phys. 11 (2014) 075 .

[23] D. Marques and C. A. Nunez, J. High Energy Phys. 10 (2015) 084.

[24] O. Hohm, S. K. Kwak, and B. Zwiebach, J. High Energy Phys. 09 (2011) 013.

[25] A. Sen, Phys. Lett. B 271, 295 (1991).

[26] J. Polchinski, Phys. Rev. Lett. 75, 4724 (1995).

[27] P. Meessen and T. Ortin, Nucl. Phys. B541, 195 (1999).

[28] D. J. Gross and E. Witten, Nucl. Phys. B277, 1 (1986).

[29] M. T. Grisaru and D. Zanon, Phys. Lett. B 177, 347 (1986).

[30] M. D. Freeman, C. N. Pope, M. F. Sohnius, and K. S. Stelle, Phys. Lett. B 178, 199 (1986).

[31] G. Policastro and D. Tsimpis, Classical Quantum Gravity 26, 125001 (2009).

[32] J. T. Liu and R. Minasian, Nucl. Phys. B874, 413 (2013).

[33] R. Minasian, T. G. Pugh, and R. Savelli, J. High Energy Phys. 10 (2015) 050.

[34] H. Razaghian and M. R. Garousi, Phys. Rev. D 97, 106013 (2018).

[35] J. Maharana and J. H. Schwarz, Nucl. Phys. B390, 3 (1993).

[36] M. Fukuma, T. Oota, and H. Tanaka, Prog. Theor. Phys. 103, 425 (2000). 